\section{BRONCHOGENIC CYSTS OF THE MEDIASTINUM}

During a 25-year period 69 patients whose ages ranged from 1 day to 64 years were treated for bronchogenic cyst of the mediastinum. The male-to-female sex ratio was 1:0.76. The cysts were symptomatic in $63.7 \%$, compressive in $43.4 \%$, and life threatening in $2.8 \%$ of cases. Symptoms and signs of compression were more frequent in infants and children than in adults. Such symptoms and signs were more dependent on the location of the cyst than on its volume. The preoperative diagnosis was wrong in $16 \%$ of cases. The cysts were approached through thoracotomy in 67 cases, including one conversion from thoracoscopy, and through cervicotomy and mediastinoscopy in one case each. The cysts opened into the respiratory tract in five cases. No communication with the esophageal lumen was observed. The cystic contents were apparently infected in three cases, but samples remained sterile at culture. There was one hospital death caused by a centrally located compressive cyst that was undiagnosed at thoracotomy. The postoperative morbidity rate was $13.4 \%$. There were no further symptoms after operation in children, but five adults reported continuing pain or dyspnea. Resection of bronchogenic cysts is recommended because of uncertainties in diagnosis and in evolution. (J THORAC CARDIOvaSC SURG 1995;109:1003-10)

Michel E. Ribet, MD, Marie C. Copin, MD, and Bernard Gosselin, MD, Lille, France
B ronchogenic cysts of the mediastinum are congenital abnormalities that can occur in infants, children, and also adults. Though benign by nature and sometimes asymptomatic, they can produce compression or irritation of adjacent structures, causing symptoms and complications. The purpose of this retrospective study was to define the clinical manifestations of bronchogenic cysts and their locations and to discuss the indications for their treatment, following recent debates on the subject. ${ }^{1-3}$

\section{Patients and methods}

During a 25-year period (1967 to 1993) 24 infants and children whose ages ranged from 1 day to 15 years and 45 adults whose ages ranged from 15 to 64 years were treated at one institution. Clinical records and histopathologic reports were available in all cases. The mean postoperative follow-up period was 4.2 years (extremes 1 to 14) before the patients were lost to follow-up.

Infants and children. There were 12 boys and 12 girls. Seventeen had symptoms at the time of operation. Standard posteroanterior radiographs were done in all cases

From the University of Lille, Lille, France.

Received for publication April 27, 1994.

Accepted for publication Oct. 13, 1994.

Address for reprints: M. E. Ribet, MD, Hôpital Calmette, F 59037 Lille Cedex, France.

Copyright (C) 1995 by Mosby-Year Book, Inc.

0022-5223/95\$3.00+ $0 \quad \mathbf{1 2 / 1 / 6 1 2 7 9}$ and lateral radiographs were done in 22 cases with a barium swallow in 11 cases, excluding two patients with respiratory distress who were immediately intubated and placed on mechanical ventilation at admission to the hospital. Computed tomography was available for six patients. Fiberoptic tracheobronchoscopy was done in seven patients.

The operative approach consisted of posterolateral (14 cases), anterolateral submammary (3), or lateral axillary (16) thoracotomy and of cervicotomy in 1 case.

Adults. There were 27 men and 18 women. Twentyseven had symptoms at the time of operation. Standard posteroanterior and lateral radiographs were done in all cases, with a barium swallow in 36 cases. Computed tomography was available for 17 patients and magnetic resonance imaging for 1 patient. Fiberoptic esophagoscopy was done in 7 patients.

The operative approach consisted of posterolateral (14 cases), anterolateral submammary (22), or lateral axillary (8) thoracotomy and of mediastinoscopy in 1 case. One lateral thoracotomy immediately followed an incomplete resection through video-assisted thoracoscopy.

During the same period, five adult patients, two men and three women whose ages ranged from 48 to 54 years, refused operation for what we considered bronchogenic cysts. One patient had symptoms and had had several episodes of bronchitis since childhood; the radiographs showed a left juxtahilar mass. One had had just one episode of bronchitis associated with a left juxtahilar mass. Three were free of symptoms, one with an intertracheoesophageal mass, one with a right juxtahilar mass, and one with a left subhilar mass. 


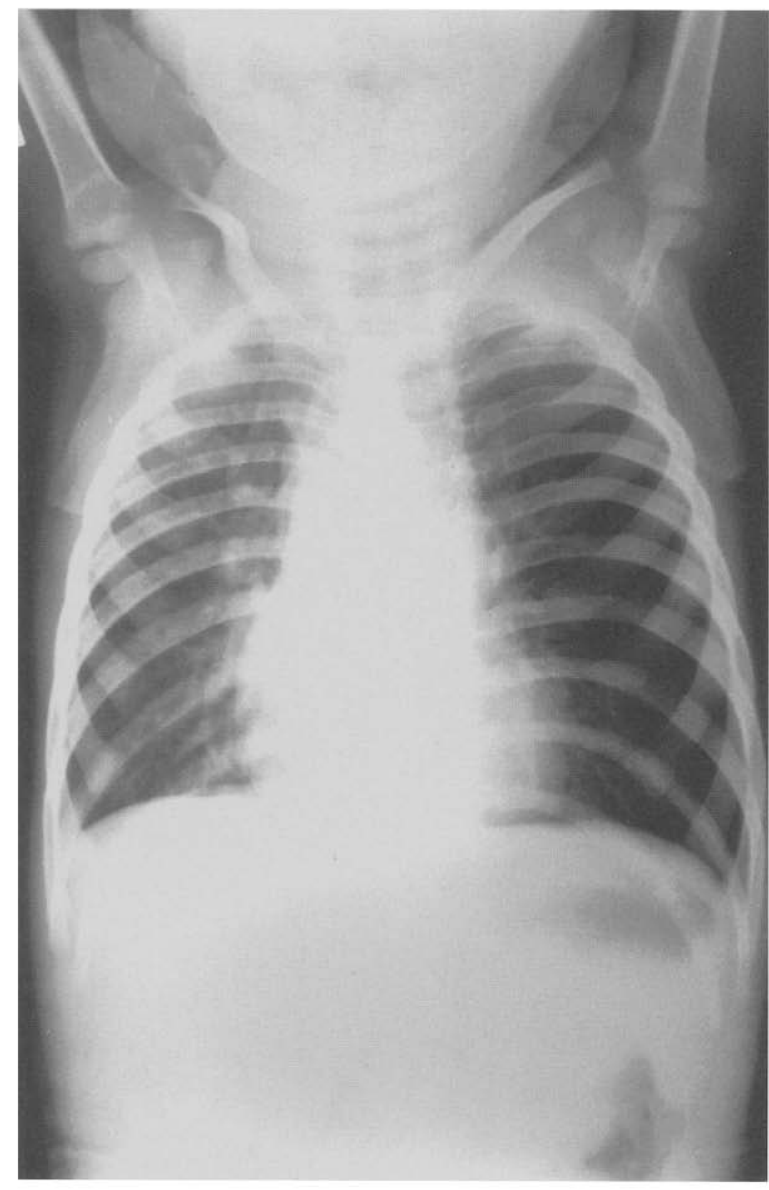

Fig. 1. Standard posteroanterior radiograph may not show abnormal shadow: signs of obstructive emphysema exist on left lung.

\section{Results}

Infants and children. As noted in Table I, the symptoms were unique or definitely prevalent in infants and children. Standard posteroanterior radiographs were insufficient to reveal an abnormal shadow in four children with symptoms, including two with respiratory distress, because the mass did not project beyond the limits of the mediastinum or was concealed behind the lung hilum, the heart, or the diaphragmatic dome (Fig. 1). A lateral radiograph was insufficient in one case only (Fig. 2) in which a barium swallow subsequently showed a print of the mass (Fig. 3).

Locations of the cysts are summarized in Fig. 4. There were radiologic signs of compression on the trachea (5 cases), the left main bronchus (5), the right main bronchus (2), the intermediate bronchus (3), and the esophagus (1). Endoscopic examina-
Table I. Symptoms observed before and after the age of 15 years

\begin{tabular}{lccc}
\hline & $\begin{array}{c}\text { Symptoms in 24 } \\
\text { infants and children } \\
\text { (unique or prevalent) }\end{array}$ & \multicolumn{2}{c}{$\begin{array}{c}\text { Symptoms in } 45 \\
\text { adults }\end{array}$} \\
\cline { 3 - 4 } & $6(25 \%)$ & $2(4 \%)$ & $5(11 \%)$ \\
Dyspnea & $4(16 \%)$ & $5(11 \%)$ & $1(2 \%)$ \\
Cough & $1(4 \%)$ & $9(18 \%)$ & $1(2 \%)$ \\
Pain & $4(16 \%)$ & $7(14 \%)$ & $1(2 \%)$ \\
Bronchopulmonary & & & Associated \\
$\quad$ infection & & $4(8 \%)$ & $2(4 \%)$ \\
Dysphagia & & & $2(4 \%)$ \\
Heartburn & $2(8 \%)$ & & \\
Respiratory distress & $7(29 \%)$ & $18(40 \%)$ & \\
No symptoms & & \multicolumn{2}{l}{} \\
\hline
\end{tabular}

tions showed bulging of the tracheal or bronchial wall with no specific sign. No transparietal puncture procedures were done.

The preoperative diagnosis was uncertain in two cases of respiratory distress with no evident mass on the posteroanterior radiographs and the diagnosis was wrong in one case thought to be a neurogenic tumor because of its laterovertebral location and radiologic density.

Complete excision of the cyst was done in 22 cases and was often made easier by an aspiration of the contents to allow a safe dissection of the deep adhesions to adjacent structures. In three cases of symptomatic and compressive cysts a fistula with the trachea (1 case), main bronchus (1), and intermediate bronchus (1) was sutured.

One 7-month-old child, who had been operated on 3 weeks before through a thoracotomy in an other institution, was admitted to our institution because of recurring dyspnea and tracheal compression. He underwent reoperation through a left cervicotomy and a cystic remnant was discovered that opened into the trachea through a $12 \mathrm{~mm}$ orifice. The cystic wall was resected, the trachea was sutured, and the suture was reinforced with prethyroid muscles. The symptoms disappeared.

One excision was done during an immediate second-stage operation on an 8-month-old male child admitted to the hospital with respiratory distress with hyperinflation of the left lung. A first thoracotomy permitted an ill-advised resection of the left thymic lobe. After extubation and a few hours of improvement the situation worsened and a new thoracotomy permitted discovery and excision of a deeply situated intertracheoesophageal cyst just above the bifurcation. The recovery was definitive. 


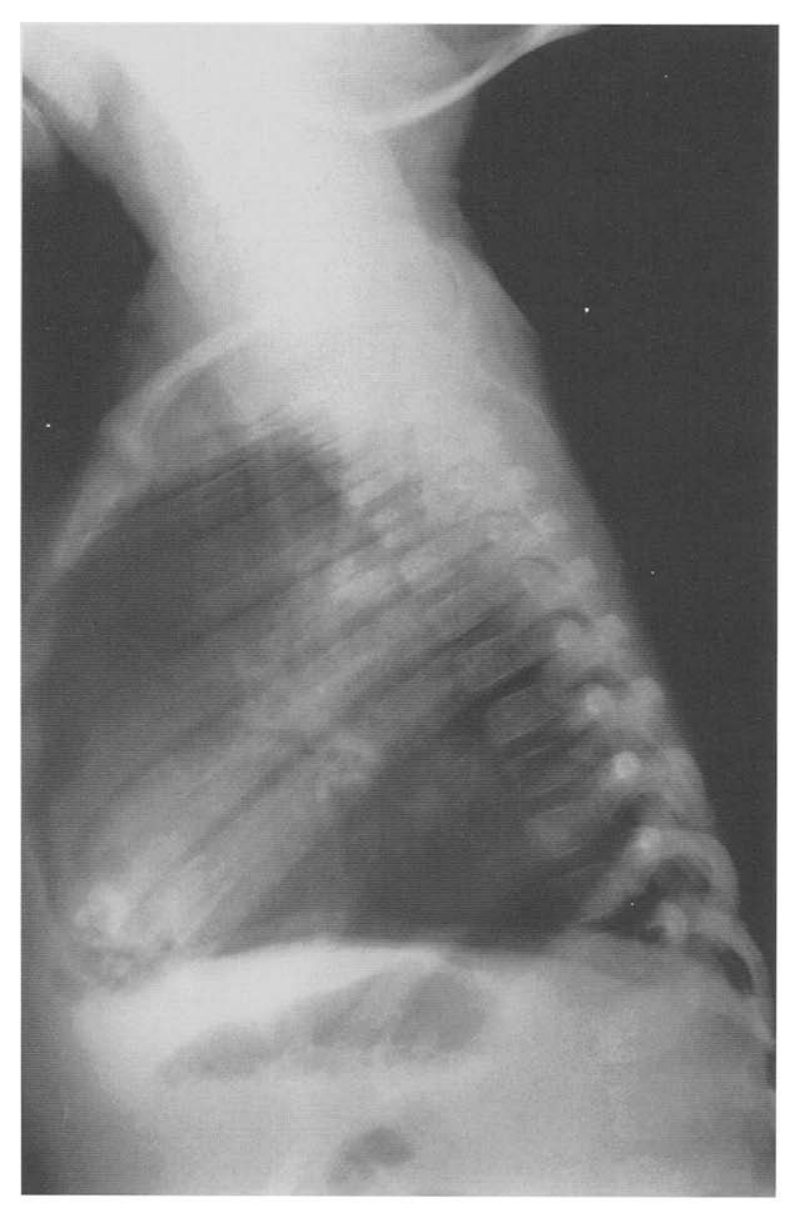

Fig. 2. Lateral radiograph shows mass in almost all cases (same patient as in Fig. 1).

One cyst lay within the esophageal wall and was excised in the submucosal plane. One operative wound of the right main bronchus membranous wall was sutured with no complication.

The excision was incomplete in one case of left paratracheal cyst in which a patch of remaining cystic mucosa was scraped and cauterized with a $2 \%$ silver nitrate solution.

The excision was not done in a newborn male infant who was operated on while in respiratory distress on the first day of life with the diagnosis of tracheobronchial compression. A large patent ductus arteriosus was discovered and thought to be responsible for the compression. This was sectioned and sutured. The formerly dense left lung immediately aerated but the child could not be durably weaned from the ventilator. The left lung progressively became overinflated and the

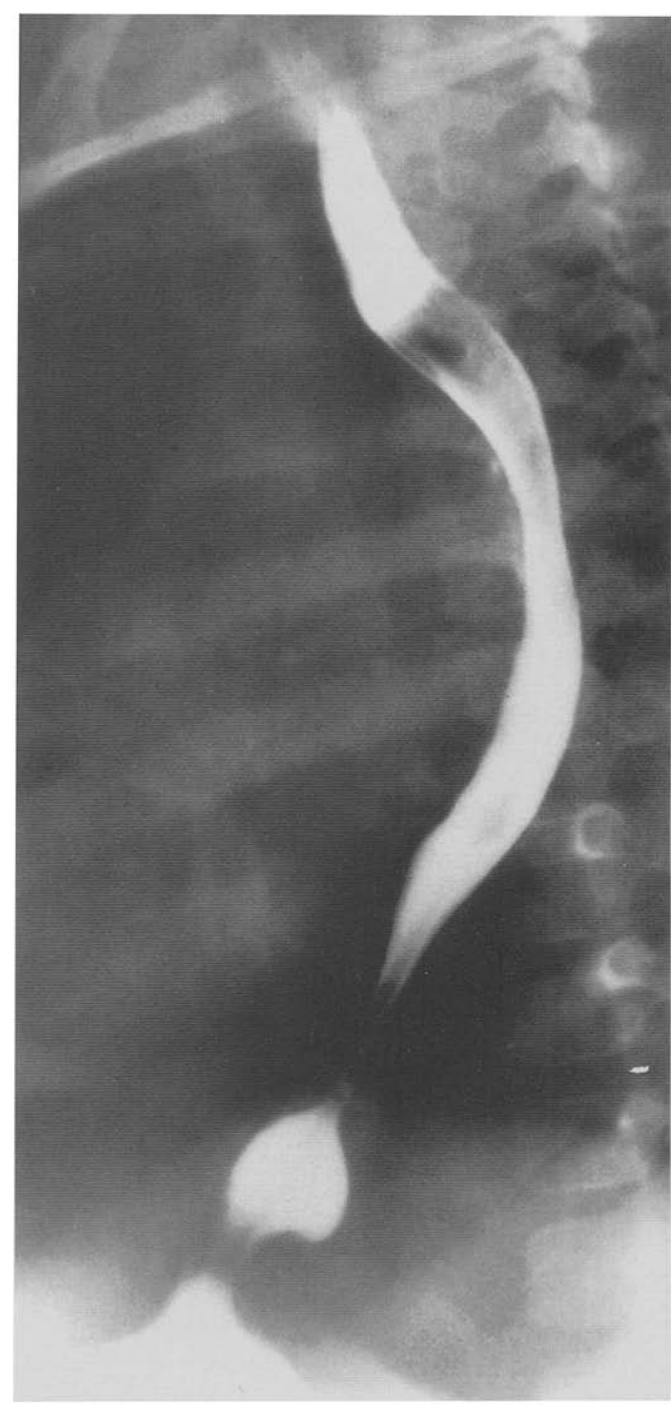

Fig. 3. Esophagogram often outlines print of cyst.

patient died on postoperative day 19 of a tension pneumothorax. The autopsy discovered an ignored $3 \times 1.5 \mathrm{~cm}$ cyst adjacent to the left main bronchus (Figs. 5 and 6).

The diameter of the cysts varied between 2 and 10 $\mathrm{cm}$ (mean $4 \mathrm{~cm}$ ). The mucus contents were more or less clear and fluid. Histopathologic examination showed in all cases a ciliated columnar mucosal lining, sometimes pseudostratified, with rare areas of squamous metaplasia. The submucosal layer was often infiltrated by chronic inflammatory cells. Bronchial glands, smooth muscles, and nerve tissue were also found. Islets of cartilage were observed in. eight cases. 


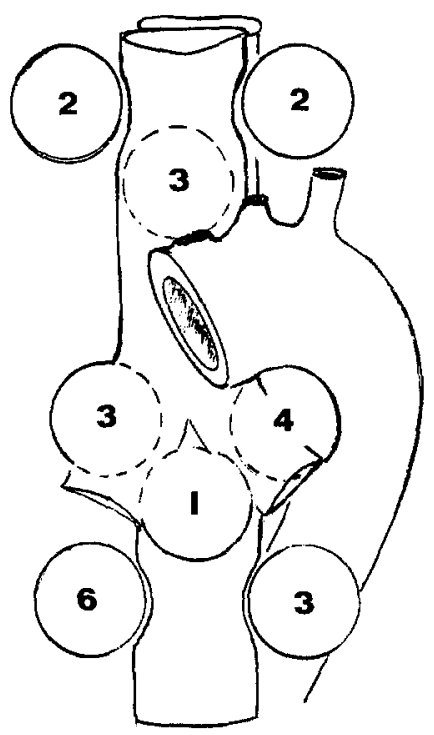

Fig. 4. Locations of cysts in infants and children. The figures indicate the number of cysts found in each location.

There was one postoperative death, which was that in the newborn infant in whom the cyst was not discovered at thoracotomy. Morbidity consisted of one pulmonary infection.

No child had any symptom during the period of follow-up. The incompletely resected cyst did not recur.

Adults. The symptoms were often associated in adults (Table I). Standard posteroanterior radiographs showed no abnormal shadow in seven adults with symptoms and a lateral radiograph did not show a shadow in one case in which a barium swallow subsequently showed the anomaly.

Locations of the cysts in adults, in contrast with children, are summarized in Fig. 7. There were radiologic signs of compression on the tracheal bifurcation (1), the intermediate bronchus (3), and the esophagus (10). Esophagoscopic examination showed regular rising of the mucosa, which was never subjected to biopsy.

The preoperative diagnosis was wrong in seven cases: four cysts were mistaken for leiomyomas because of their images in the esophageal lumen; two were mistaken for neurogenic tumors because of their laterovertebral, though anterior to the foramen, location; and one was mistaken for a carinal lymph node mass in a coal miner with silicosis. Inversely and apart from this population, an erroneous diagnosis of cyst was proposed in three masses with low Housefield units: one cystic neuro- genic tumor, one benign Castleman lymphoma, and one hemolymphangioma.

A complete excision of the cyst was done in 43 cases. In one case of symptomatic cyst a fistula with the trachea was closed. A thoracoscopy was converted into an axillary thoracotomy because of adhesions between the cyst and the tracheal bifurcation. Two pulmonary lobectomies were necessitated by hilar cysts creeping in between the lobar vessels and bronchi. Seven cysts dissociated the muscular fibers of the esophagus or lay within its wall. These were all excised without opening the esophageal lumen. One operative wound of the trachea membraneous wall was sutured with no complication.

The excisions were incomplete in one case of paratracheal and one case of hilar cyst, in which a small patch of mucosa was left in place, which was scraped and cauterized.

A carinal cyst, mistaken for lymph nodes, was submitted to mediastinoscopy: $150 \mathrm{ml}$ of mucus was aspirated and biopsy studies proved the bronchogenic cyst.

The diameter of the cysts varied between 2 and $15 \mathrm{~cm}$ (mean $4.5 \mathrm{~cm}$ ). The mucus contents looked purulent in three cases, but were sterile at culture. Histopathologic findings were the same as observed in children, with more frequent areas of squamous metaplasia and islets of cartilage in 13 cases. Acute inflammation of the cystic wall was present in the three cases with purulent contents.

Morbidity consisted of four pulmonary infections, three cases of medically cured chylothorax, and one case of persistent phrenic paresia.

Five patients reported persisting pain or dyspnea, or both, during the follow-up period: three had had symptoms and two had not had symptoms before operation. The image of the carinal punctured cyst, decreased by one third, was stable and the cyst remained asymptomatic after 2 years. The two incompletely resected cysts did not recur.

Follow-up was obtained from two of the five patients who had refused operation. Both had been free of symptoms at first examination. One patient with an intertracheoesophageal mass remained free of symptoms after 5 years and one patient with a subhilar mass died after 15 years of follow-up of a "generalized malignancy of unknown origin."

\section{Discussion}

Bronchogenic cysts originate from the ventral part of the endodermic primitive foregut. ${ }^{4}$ The respiratory budding arises between days 20 and 40 of 


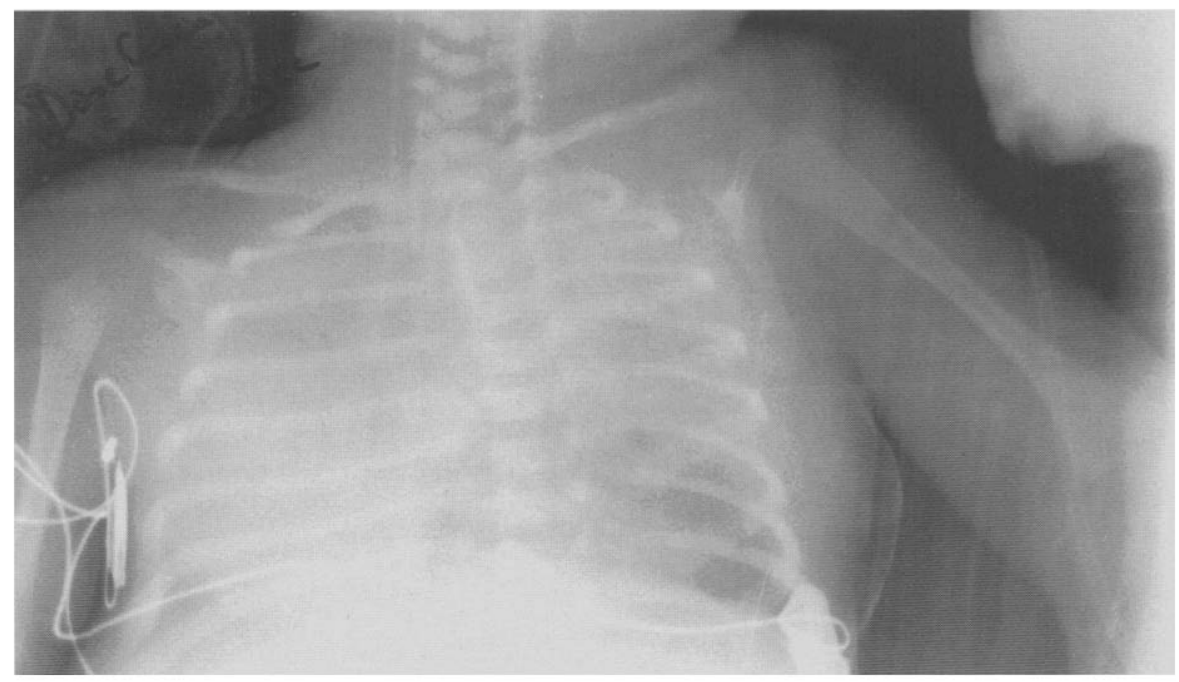

Fig. 5. Radiograph of newborn infant with respiratory distress. Diagnosis was tracheobronchial compression of undetermined cause.

gestation and the cleavage between the respiratory tract and the digestive tube occurs on day 28. Both respiratory and digestive mucosae are columnar and ciliated at that time: not before the fourteenth week squamous epithelium spreads from the middle third of the esophagus to its extremities. Meanwhile, during the sixth week, the mesoderm grows to produce the esophageal muscular layers (ninth to twelfth weeks), the cartilage (tenth week), the glands (eleventh week), and the lung alveoli (twenty-fourth week). The embryogenesis thus explains that cysts lined with a respiratory-type mucosa are apt to develop not only along the trachea, along the bronchi, and in the lung, depending on the moment of their formation, but also along the esophagus, including below the diaphragm, as the esophagus grows longer caudally during the second month of gestation. $^{5}$

This accounts also for the difficulties sometimes encountered by surgeons and pathologists in differentiating the tracheobronchial or esophageal origin of these malformations. The embryogenesis explains that all the components of the normal tracheobronchial wall are not always present in the cystic structure, particularly the cartilage: the presence of this component depends on the fusion of mesoderm fragments to the mucosal cystic formation. Cartilage was found in $32.7 \%$ of the cysts adjacent to the tracheobronchial tree and in $25 \%$ of the cysts connected with the esophagus in our population and in

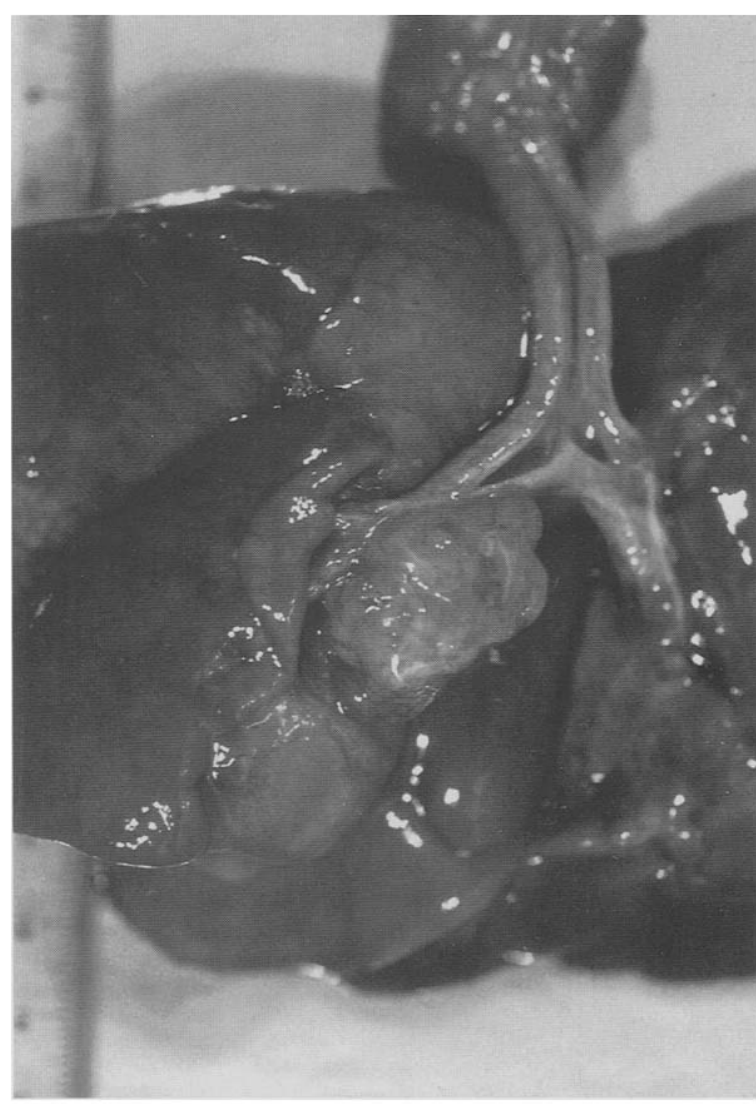

Fig. 6. Same patient as in Fig. 5. Section-suture of large patent ductus arteriosus improved situation with no definitive cure. Death occurred on postoperative day 19 and autopsy discovered ignored compressive cyst. 


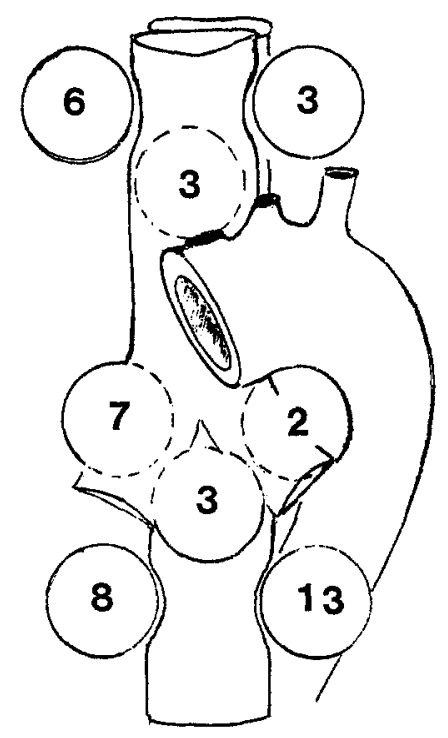

Fig. 7. Locations of cysts in adults. The figures indicate the numbers of cysts found in each location.

only 1 case out of 12 in a published series. ${ }^{6}$ There was no difference between the two locations concerning mucosa and other components. It is also admitted there can be no cartilage in lung bronchogenic cysts and cartilage islets have been observed in congenital esophageal stenosis. Thus there is a tendency to unify the cysts according to their columnar ciliated mucosal lining, whatever their locations. ${ }^{7}$ Nevertheless, considering their connections with the esophagus, they have been divided into inclusion cysts that spread out the muscle fibers, duplication cysts that lie under the two muscle layers, and true bronchogenic cysts with cartilage. ${ }^{8}$ All these cysts come from a ventral anomaly of the foregut and the subvarieties make no practical difference. Although they must be distinguished from the dorsal anomalies of the foregut and chorda, which are associated with vertebral and meningeal anomalies, producing neuroenterogenous cysts, and also from gastroenterogenous cysts, which are lined with gastric mucosa, ${ }^{9}$ bronchogenic cysts are part of the foregut cysts group.

The contents of bronchogenic cysts are mucoid, clear or yellow, sometimes turbid or purulent, and rarely hematic.

Bronchogenic cysts account for $6 \%$ to $15 \%$ of primary mediastinal masses; $8 \%$ in our experience. The male:female sex ratio varies in the literature and was 1:0.76 in our series. It is difficult to appreciate the exact prevalence of the cysts: the advanced age of some patients proves that some cysts probably remain forever silent. The comparison of the mean diameters of the cysts before and after the age of 15 years is in favor of a very slow progression in volume with age.

The cysts are observed more often on the right side of the mediastinum, perhaps because they are more easily concealed on the left side. Their topography appears to be more important than their volume, as concerns risks of symptomatic compression: $57 \%$ for paratracheal locations, $68 \%$ at the bifurcation and hilum level, and $16 \%$ below the hilum. It is clear that the first two sites are more apt to have compression: there the trachea, main bronchi, great vessels, arterial ductus, and esophagus encompass the cysts, which have much more free space under the hilum. This may account for the fact that in children cysts are more often symptomatic $(70.8 \%)$, because $75 \%$ are situated above the hilum and at the level of the hilum, and that they are less symptomatic in adults (60\%), because $53 \%$ are situated above the hilium or at the level of the hilium. An important obstruction of the respiratory tract is particularly apt to occur with a cyst that does not bulge under the mediastinal pleura, especially on the left side where it is covered by the aortic arch. It is also on the left side that the main bronchus is longer, narrower, and more easily compressed by a cyst contained by the aorta and ductus. The relatively large mean diameter of the cysts before 5 years of age, $3.4 \mathrm{~cm}$ in our population, added to the narrowness and softness of the trachea and bronchi in infants and young children, explains that during that period of life there is an almost complete correlation between compression and symptoms (dyspnea and bronchopulmonary infection): 14 cases out of 15 (Fig. 8).

Compression can provoke respiratory distress. ${ }^{10}$ More rarely it can affect caval ${ }^{11}$ or pulmonary circulation. $^{12,13}$ Esophageal compression is symptomatic in adults. After the age of 5 years, symptoms are no longer always caused by a mechanical effect of the cyst. Pain then becomes the most frequent symptom, perhaps caused by irritation of the pleura. At the same time, asymptomatic cysts are more frequent (Fig. 8). In our population, compressive symptomatic cysts comprised $79 \%$ of those in children and $29 \%$ of those in adults. Noncompressive symptomatic cysts comprised, respectively, $0 \%$ and $31 \%$. Asymptomatic cysts comprised, respectively, $29 \%$ and $40 \%$, including one radiologically compressive mass in each group.

If there is no discussion concerning the treatment 


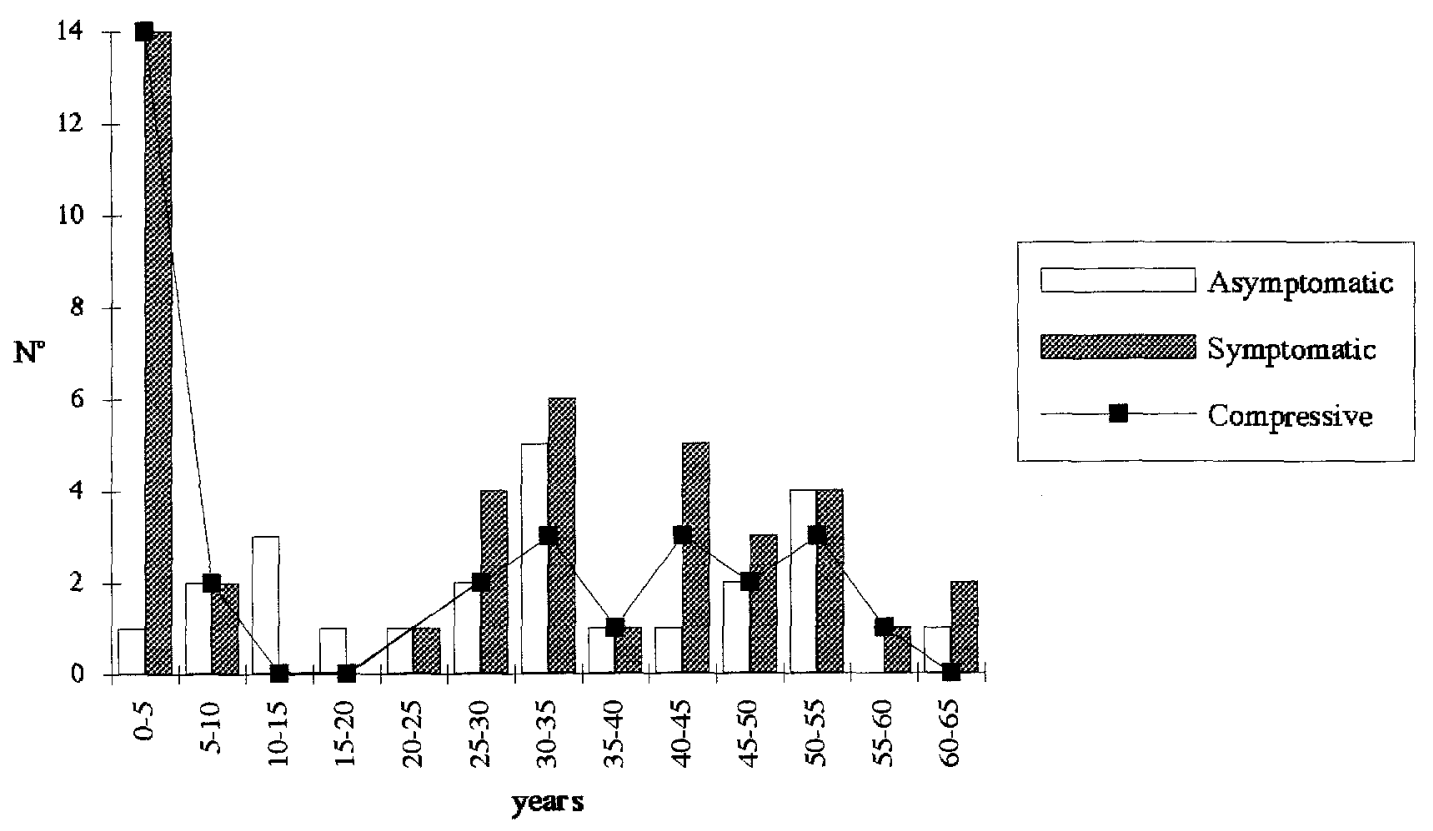

Fig. 8. Symptoms and signs of compression according to age.

of a compressive symptomatic cyst, which can obstruct adjacent organs and hamper their development in infants and children, the relative rarity of compression and complications at a later age, the incertitude concerning the causes of symptoms, a small volume, and the percentage of asymptomatic cysts are arguments for therapeutic abstention. But in no case can a patient, or his or her parents, be completely reassured about the future. The diagnosis of a mediastinal mass, despite modern imaging modalities including computed tomography and, still better, magnetic resonance imaging, ${ }^{14}$ is not always certain and though our own errors did not include any malignancies, these errors concerned $16 \%$ of our patients. Computed tomography, although accurate in determining the anatomy of a mediastinal mass when it is connected with the tracheobronchial tree, is less precise concerning its relation to the esophageal wall. As for the nature of the mass, density and heterogenous aspect can be sources of errors: some solid edematous tumors can be mistaken for cysts. Indeed, from a practical and economic point of view, standard posteroanterior and lateral radiographs, with a barium swallow, are sufficient for the diagnosis in $80 \%$ of cases and at all ages.

Furthermore, the presence of an abnormal mediastinal mass demands regular follow-up and the knowledge of a thoracic abnormality can produce anxiety in childrens' parents and secondary symp- toms in adult patients, as we observed in four of these adult patients after a fortuitous discovery of the cyst.

Video-assisted thoracoscopic surgery might be better accepted by those patients who refuse thoracotomy. ${ }^{15}$ In no case, however, should their security be at risk because of such an offer from the surgeon. We believe that indications for operation are not changed by new techniques and that the dissection and excision of a bronchogenic cyst, even when it shows well under the mediastinal pleura, can be uneasy underneath. The need for conversion to a thoracotomy is possible. A retrospective review of the operative records showed that a central topography, adhesions, and communications of the cysts with the tracheobronchial tree would have made a thoracoscopic approach hazardous in $30 \%$ of the children and $11 \%$ of the adults.

Complete resection is the best treatment. Transparietal, ${ }^{16}$ transbronchial, ${ }^{17}$ or mediastinoscopic $^{18}$ puncture and aspiration are exceptionally preferred as temporary measures in case of acute compression or in selected cases. A deep exploration with careful dissection of the mediastinal structures is necessary in case of compression of doubtful origin, so that the case is not confounded by another possible cause such as a patent ductus arteriosus or a large thymus and so that an unnecessary pulmonary resection is avoided when an obstructive emphysema is mistaken for a giant lobar emphysema, 
which may present the same operative aspect: a centrally located cyst may be difficult to discover from a left-sided approach and most respiratory obstructions happen on the left side. Even though a right approach of the central mediastinum is easier, it is neither logical nor secure when there are signs of left pulmonary obstruction.

Operative difficulties from adhesions to adjacent organs are possible, but the most important point is the dissection of the cyst from the tracheobronchial wall, where a thin common membrane or an orifice may exist. In difficult cases, a small patch of cystic wall may be left in place after destruction of the mucosal lining to avoid recurrence. The dissection of the esophageal mucosa is easier: except in case of an unfortunate preoperative endoscopic biopsy, there is no communication between the cyst and the esophageal lumen.

Postoperative mortality is exceptional and morbidity is acceptable. The results are good but symptoms may persist or even appear in adults.

\section{REFERENCES}

1. St. Georges R, Deslauriers J, Duranceau A, et al. Clinical spectrum of bronchogenic cysts of the mediastinum and lung in the adult. Ann Thorac Surg 1991;52:6-13.

2. Bolton JWR, Shahian DM. Asymptomatic bronchogenic cysts: what is the best management? Ann Thorac Surg 1992;53:1134-7.

3. Suen HC, Mathisen DJ, Grillo HC, et al. Surgical management and radiological characteristics of bronchogenic cysts. Ann Thorac Surg 1993;55:476-81.

4. Gray SW, Skandalakis JE. Embryology for surgeons. Philadelphia, London, Toronto: WB Saunders, 1973: 81-5, 311-4.

5. Coselli MP, De Ipolyi P, Bloss RS, Diaz RF, Fitzgerald JB. Bronchogenic cysts above and below the diaphragm: report of eight cases. Ann Thorac Surg 1987;44:491-4.
6. Sirivella S, Ford WB, Zikria EA, Miller WH, Samadini SR, Sullivan ME. Foregut cysts of the mediastinum. J Thorac Cardiovasc Surg 1985;90:776-82.

7. Postlethwait RW. Benign tumors of the esophagus. Surg Clin North Am 1983;63:925-31.

8. Salo JA, Ala-Kulju KV. Congenital esophageal cysts in adults. Ann Thorac Surg 1987;44:135-8.

9. Rodgers BM, Harman PK, Johnson AM. Bronchopulmonary foregut malformation: the spectrum of anomalies. Ann Surg 1985;203:517-24.

10. Eraklis AJ, Griscom NT, McGovern JB. Bronchogenic cysts of the mediastinum in infancy. $\mathrm{N}$ Engl $\mathrm{J}$ Med 1969;281:1150-5.

11. Miller DC, Walter JP, Guthaner DF, Mark JBD. Recurrent mediastinal cyst: cause of bronchial obstruction and compression of superior vena cava and pulmonary artery. Chest 1978;74:218-20.

12. Selke AC, Belin RP, Durnin R. Bronchogenic cyst in association with hypoplasia of the left pulmonary artery. J Pediatr Surg 1975;10:541-3.

13. Worsnop CJ, Teichtahl H, Clarke CP. Bronchogenic cyst: a cause of pulmonary artery obstruction and breathlessness. Ann Thorac Surg 1993;55:1254-5.

14. Brasch RC, Gooding CA, Lallemand DP, Wesberg GE. Magnetic resonance imaging of the thorax in childhood. Radiology 1984;150:463-7.

15. Acuff TE, Mack MJ, Ryan WH, Bowman RT, Douthit MB. Thoracoscopic excision of bronchogenic cysts. Ann Thorac Surg 1993;55:196-20.

16. Zimmer WD, Kamida CB, McGough PF, Rosenow EC. Mediastinal duplication cyst: percutaneous aspiration and cystography for diagnosis and treatment. Chest 1986;90:772-3.

17. Schwartz AR, Fishman EK, Wang KP. Diagnosis and treatment of a bronchogenic cyst using transbronchial needle aspiration. Thorax 1986;41:326-7.

18. Ginsberg RJ, Atkins RW, Paulson DL. A bronchogenic cyst successfully treated by mediastinoscopy. Ann Thorac Surg 1972;13:266-8. 